\title{
¿QUIÉNES ESTUDIAN SOCIOLOGÍA EN LA UNIVERSIDAD DE COSTA RICA? ${ }^{1}$
}

\section{WHO STUDIES SOCIOLOGY AT THE UNIVERSITY OF COSTA RICA?}

\author{
Irina Sibaja López*
}

\section{RESUMEN}

En el presente artículo se expone una parte de los principales resultados de la Caracterización del Perfil del Estudiantado Activo en la Escuela de Sociología de la Universidad de Costa Rica: su relevancia académico-administrativa. Se buscó recuperar un perfil amplio, más allá de las concepciones tradicionales, por lo que se incluyeron cinco dimensiones de análisis que son la demográfica, la socioeconómica, la académica, las aspiraciones profesionales y el proyecto de vida. En el artículo se expone la fase cuantitativa, que consistió en la aplicación de un cuestionario digital autoadministrado. Como principales conclusiones, se encontró a una población joven, costarricense, soltera, residente del Gran Área Metropolitana y dependiente económicamente de terceros. Para un amplio número de participantes, la Sociología no fue su primera opción de carrera universitaria, pero decidieron continuar con esta, donde su principal aspiración profesional es encontrar trabajo estable en Sociología, como proyecto de vida vinculado a la carrera en el corto y mediano plazo.

PALABRAS CLAVE: ESTUDIANTE UNIVERSITARIO * CALIDAD DE LA EDUCACIÓN * PROFESIÓN *
ENSEÑANZA SUPERIOR

\section{ABSTRACT}

This article shows a section of the main results of the Characterization of the Profile of Active Students in the School of Sociology of the University of Costa Rica: its academicadministrative relevance. It was sought to recover a broad profile, beyond traditional conceptions, so that five dimensions of analysis were included, which are demographic, socioeconomic, academic, professional aspirations and the life project. This article describes the quantitative phase, which consisted in the application of a self-administered digital questionnaire. For the main conclusions, a young, Costa Rican, single population,

$1 \quad$ El presente artículo expone algunos de los resultados del proyecto de investigación denominado "Caracterización del perfil del estudiantado activo en la Escuela de Sociología de la Universidad de Costa Rica: su relevancia académico-administrativa", código 211-B8251; inscrito en la Escuela de Sociología de la Universidad de Costa Rica. 
resident of the Greater Metropolitan Area, and economically dependent on others was found. For many participants, Sociology was not their first university career option, but many decided to continue with it, while others asked for a transfer. For those who continued, their most recurrent professional aspiration was to find a stable and Sociologyrelated work, as a life project, linked to the career, in the short and medium term.

KEYWORDS: UNIVERSITY STUDENTS * EDUCATIONAL QUALITY * PROFESSIONS * HIGHER EDUCATION

\section{INTRODUCCIÓN: PUNTO DE PARTIDA}

Caracterizar a la población estudiantil de una unidad académica, es sin duda un requerimiento indispensable para la toma de las decisiones, tanto académicas como administrativas. El presente artículo da cuenta de parte de los resultados de un proyecto de investigación realizado por la autora e inscrito en la Vicerrectoría de Investigación, con el cual se busca contribuir en esta línea y brindar algunas de las principales características del perfil del estudiantado activo que no ha concluido el bachillerato y la licenciatura, pero que tienen interés en finalizar la carrera en la Escuela de Sociología de la Universidad de Costa Rica para el periodo 2018, de acuerdo al Plan de Estudios de Bachillerato (2016) y Plan de Estudios de la Licenciatura (2018).

Ello como respuesta a la necesidad de saber quiénes son y qué características tienen; así como destacar la importancia de este tipo de caracterizaciones en la toma de decisiones fundamentadas, desde un conocimiento preciso de la población estudiantil y teniendo en consideración elementos que están involucrados en su formación a lo largo de una carrera universitaria.

De ahí la importancia de contar con información contextualizada. La planificación es de suma relevancia en la cotidianeidad de las unidades académicas, y la misma se torna difícil de realizar sin tener pleno conocimiento del estudiantado, y de su distribución en los diferentes niveles de la carrera.

Lo anterior se propicia a partir de un mejor diálogo con el estudiantado, lo que genera procesos de vinculación y orientación más precisos y coincidentes con su realidad y con sus necesidades. Ello, con el fin de garantizar un acompañamiento adecuado en su proceso formativo.
Por tanto, se trabajó en desarrollar una caracterización amplia del perfil, que abarcara las tradicionales dimensiones, tales como, la demográfica, la socioeconómica, la académica, y que, además, incorporara las aspiraciones profesionales $y$ el proyecto de vida; elementos que brindaron un panorama integral del estudiantado.

Con base en De Sousa Santos (2007), lo mencionado adquiere sentido cuando se invita a pensar el conocimiento universitario contextualizado, determinado por el diálogo constante entre el personal investigador, docente $y$ el estudiantado, donde cada uno de estos grupos juega un rol esencial.

Por estas razones, se buscó caracterizar un perfil amplio del estudiantado, comprendiendo que:

Las instituciones de educación superior enfrentan el reto de ofrecer a sus comunidades estudiantiles ambientes educativos, en los que se garanticen condiciones favorables para el desarrollo humano y el bienestar de las personas. Esta misión difícilmente podría ser abordada sin un proceso previo y continuo de comprensión y acercamiento a la realidad de los grupos humanos que ingresan a las universidades (Centro de Investigaciones, Departamento de Bienestar Universitario, 2005, p. 11).

Lo anterior recuerda que la institución y sus unidades académicas deben asegurar la enseñanza de sus estudiantes como uno de sus principales propósitos, desde su conocimiento contextualizado. 


\section{METODOLOGÍA: EL CAMINO RECORRIDO}

El estudio se caracterizó por ser descriptivo y exploratorio, privando el detalle y la exposición amplia de los datos del estudiantado, la cual se realiza desde una construcción teórica $y$ de los antecedentes, a partir de la conceptualización de perfil y de su importancia en las carreras universitarias.

Al no contarse con ningún estudio de naturaleza similar con la mayor parte de la población estudiantil en la Escuela de Sociología ${ }^{2}$ de la Universidad de Costa Rica, la investigación se desarrolló desde una metodología mixta, donde la articulación entre lo cuantitativo y cualitativo permite profundizar en las dimensiones que se describirán más adelante. Sin embargo, en el presente artículo solo se expondrán los principales resultados obtenidos de carácter cuantitativos.

En la primera fase, se propuso trabajar desde una metodología cuantitativa que permitiese recuperar aspectos generales de las dimensiones, para luego profundizar en aquellos elementos de mayor interés. Se utilizó como técnica el cuestionario digital auto-administrado. Se parte de la concepción de que la metodología constituye una manera de pensar la realidad social y de estudiarla (Strauss y Corbin, 2002), de aquí que no solo implique el uso de ciertos métodos, sino también la forma en que se interpretan los datos. No obstante, la solución más adecuada está sujeta a las necesidades del problema de investigación. Por ello, se profundizará en las decisiones tomadas al respecto, en función del camino recorrido durante el proceso.

Se procuró caracterizar un perfil amplio del estudiantado, lo que implicó no solo incluir dimensiones como la demográfica y la socioeconómica, que son las que tradicionalmente se incluyen en este tipo de estudios ${ }^{3}$, y que dan cuenta del

2 Esto al tener en consideración el trabajo realizado por la Dra. Eugenia Molina (2016), docente de la Escuela de Sociología. Además de la investigación con estudiantes de Sociología a nivel latinoamericano de la investigadora Norma Caira y el investigador Isaías Lescher (2013).

3 Para ampliar el tema, se destacan las investigaciones a nivel nacional de Rodríguez y Rojas (2015), Cruz y Rodríguez (2013). Así como las entramado más amplio en el que se desenvuelve el estudiantado. También se propuso examinar algunos elementos de interés que refieran a lo académico, a sus aspiraciones profesionales $y$ proyecto de vida. Cada una de las dimensiones están compuestas por las siguientes variables:

$\diamond \quad$ Demográfica: interesó reunir información respecto a variables como la edad, el estado civil, la nacionalidad, el género, si tienen o no hijos, la convivencia, así como, el lugar de origen $y$ residencia.

$\diamond \quad$ Socioeconómica: se profundizó en el nivel económico y en las condiciones laborales, tanto del estudiantado como del contexto familiar, que permiten caracterizar el grupo social al que pertenecen. Ello, a partir de la recuperación de información sobre el nivel educativo de sus padres, con el fin de conocer la principal actividad y la posición que ocupan en sus respectivas labores.

$\diamond \quad$ Académica: sin duda es una dimensión relevante en términos de la elaboración del perfil del estudiantado, ya que permitió conocer el lugar que ocupan en el Plan de Estudios y la forma en que lo completan.

$\diamond \quad$ Aspiraciones profesionales: por lo general, en los perfiles, una dimensión como las aspiraciones profesionales no es común; ya que se prioriza el análisis desde elementos de carácter objetivo. De ahí que se buscó recuperar estas aspiraciones con el fin de vincularlas con su paso por la carrera. Interesó conocer la percepción del estudiantado sobre lo que espera de su futuro profesional y como lo perciben desde su posición como estudiantes de la carrera de Sociología.

$\diamond \quad$ Proyecto de vida: lo indicado para la dimensión anterior sobre la importancia de la subjetividad, también aplica para el proyecto de vida. Se incluyó para conocer las vinculaciones que la población estudiantil proyecta entre la carrera de Sociología y su vida futura.

investigaciones a nivel latinoamericano de Carreño, Micin y Urzua (2016), Garza, Contreras y Ruiz (2013) y Garay (2003). 
La población de la investigación la constituyó el estudiantado activo, tanto el de bachillerato como el de licenciatura, durante el primer ciclo del 2018 de la Escuela de Sociología. Mientras que la unidad de análisis se compone de la proporción de la población activa que no ha completado la carrera y que tiene interés en finalizarla. Se determinó que se contaba con una población activa de 970 estudiantes, de los cuales se excluyeron 23 por encontrarse graduados y graduadas, para un total definitivo de 947 estudiantes en estado activo(as) no graduado, tanto de bachillerato como de licenciatura. A las 947 personas estudiantes se les hizo llegar el cuestionario. De ellas, 629 estudiantes respondieron, 127 estudiantes indicaron no tener interés en finalizar la carrera, y, por tanto, no continuaron completando el instrumento; mientras que los 502 estudiantes restantes sí manifestaron su interés por la carrera, ya sea que en el periodo de estudio hubiesen realizado matrícula o no. Estos últimos se configuraron de forma efectiva en la unidad de análisis del proyecto. Si bien, no logró recuperarse información de la totalidad del estudiantado, se considera que los datos recuperados dan cuenta de las principales características de la población estudiantil, siendo un importante aporte a su comprensión.

De ahí la relevancia del proyecto de investigación, ya que conocer quienes intervienen en el proceso educativo, en este caso, la población estudiantil, es una necesidad para garantizar el adecuado ejercicio de la enseñanza universitaria, de la gestión administrativa y de la orientación en las unidades académicas.

\section{PRINCIPALES RESULTADOS: PERFIL DEL ESTUDIANTADO ACTIVO EN SOCIOLOGIA}

La información que se expondrá con detalle, da cuenta de los principales resultados obtenidos desde los datos recolectados a partir de las cinco dimensiones de análisis expuestas.

\section{CARACTERÍSTICAS DEMOGRÁFICAS}

Del total de 502 estudiantes que completaron el cuestionario, se encuentra que la edad de ingreso promedio a la carrera es de 33 años, siendo los 18 años la edad más frecuente. Mientras que la edad promedio actual (año 2018, momento en que se aplica el instrumento) de la población estudiantil es de 36 años, donde los 21 años se configura como la edad más recurrente. En ambos casos, la edad mínima corresponde a los 17 años y la edad máxima registrada es de 67 años al momento de ingreso y 71 años en el año 2018.

Asimismo, el $82 \%$ de la población estudiantil cuando ingresa a la carrera se ubica entre los 17 y 22 años, lo que equivale a 412 estudiantes, por su parte, el $18 \%$ restante se encuentra entre los 23 y los 67 años. Sin duda, se puede indicar que la población estudiantil se caracteriza por ser joven, principalmente en el momento de ingreso a la carrera. Situación que evidencia que la mayor parte del estudiantado ingresa a la carrera casi inmediatamente luego de finalizada la educación secundaria, sea como primera o segunda opción en el concurso a carrera.

Cabe destacar, que a pesar de que la mayor parte de la población ingresa a la carrera entre los 17 y 22 años, sin embargo, en el año 2018 , se concentra en el rango de edad entre los 23 y 28 años, lo que evidencia que la mayoría de la población no completa la carrera dentro del plazo establecido por el Plan de Estudios vigente, que comprende 4 años para el bachillerato $y$ uno más para la licenciatura.

Con respecto a la distribución por género, se encontró una prevalencia del género femenino en la carrera con un total de 289 personas, equivalente al $58 \%$. Mientras que el género masculino representa un $41 \%$, correspondiente a 206 personas, y el $1 \%$ restante corresponde a las personas que se identifican con el género no-binario (7 personas). Esta tendencia se ha registrado en la Escuela a lo largo de los últimos 5 años, situación similar en la población estudiantil general de la Universidad.

En lo que respecta al estado civil, existe una marcada prevalencia de estudiantes solteras o solteros, por encima de las personas casadas, divorciadas o en unión libre. Siendo 458 personas solteras, lo que equivale al $91 \%$ de la población de análisis. El restante $8 \%$ corresponde a los demás estados civiles ( $4 \%$ 
casados(as), 3\% unión libre y 1\% divorciados(as)). Datos que resultan coincidentes y estadísticamente significativos con la edad actual de la población estudiantil. Asimismo, del total de personas solteras, casadas y divorciadas, la mayoría se identifica con el género femenino, mientras que la mayoría de las personas en unión libre se ubican dentro del género masculino. Todas las personas del género no-binario son solteras.

En lo que respecta a la nacionalidad del estudiantado, el $98 \%$ son personas costarricenses, mientras que el $2 \%$ (6 estudiantes) corresponde a otras nacionalidades, como nicaragüense, china, guatemalteca y argentina.

$\mathrm{Al}$ consultarles si tienen hijos o hijas, se encuentra que la mayoría de la población estudiantil no tiene lo que equivale al $90 \%$ del estudiantado (454 estudiantes), por su parte, del $10 \%$ restante el $8 \%$ tiene solo un hijo o hija, $y$ el $2 \%$ tienen más de dos hijos o hijas. Destaca que de la población que sí tiene hijos o hijas, la mayoría la constituye personas del género femenino, con un total de 30 personas, en contraste con 18 personas del género masculino.

A lo anterior, se suma que el $66 \%$ del estudiantado vive con sus padres $\mathrm{u}$ otros familiares, el $11 \%$ solo o sola, el $9 \%$ con su pareja, el $8 \%$ con compañeros o compañeras de universidad y el $6 \%$ vive con amigos o amigas.

Al ser el Gran Área Metropolitana (GAM) el lugar de origen y residencia de la mayor parte de la población estudiantil, correspondiendo al $88 \%$ y al $97 \%$ respectivamente, con una mayor concentración en la provincia de San José seguida por Cartago, Heredia y Alajuela. Aunque se cuenta con población estudiantil de todas las provincias, tanto como lugar de origen $y$ como lugar de residencia, las provincias de Limón, Puntarenas y Guanacaste no agrupan un número amplio de población, ya que cuando se analizan como lugar de origen, un $12 \%$ del estudiantado proviene de ellas, mientras que, como provincias de residencia actual, solo el $3 \%$ de la población viven en ellas (ver tabla 1 ).

TABLA 1

PROVINCIA DE ORIGEN Y DE RESIDENCIA DEL ESTUDIANTADO DE LA CARRERA DE SOCIOLOGÍA UNIVERSIDAD DE COSTA RICA, 2018

\begin{tabular}{l|cc|cc}
\hline \multirow{2}{*}{ PROVINCIA } & \multicolumn{2}{|c|}{ ORIGEN } & \multicolumn{2}{c}{ RESIDENCIA } \\
\cline { 2 - 5 } & $\begin{array}{c}\text { VALORES } \\
\text { ABSOLUTOS }\end{array}$ & PORCENTAJE & $\begin{array}{c}\text { VALORES } \\
\text { ABSOLUTOS }\end{array}$ & PORCENTAJE \\
\hline San José & 245 & $49,1 \%$ & 296 & $59.1 \%$ \\
Cartago & 87 & $17.4 \%$ & 83 & $16.6 \%$ \\
Heredia & 44 & $8.8 \%$ & 61 & $12.2 \%$ \\
Alajuela & 64 & $12.8 \%$ & 48 & $9.6 \%$ \\
Limón & 27 & $5.4 \%$ & 7 & $1.4 \%$ \\
Puntarenas & 16 & $3.2 \%$ & 5 & $1.0 \%$ \\
Guanacaste & 16 & $3.2 \%$ & 1 & $0.2 \%$ \\
\hline TOTAL & 499 & $100 \%$ & 501 & $100 \%$ \\
\hline
\end{tabular}

Fuente: Elaboración propia a partir de los datos del cuestionario aplicado al estudiantado activo de la carrera de Sociología durante el ciclo I-2018.

Si bien, la mayoría vive en la GAM, se da un proceso de migración interna que se evidencia entre quienes indicaron que su provincia de origen era Alajuela, Limón, Puntarenas y Guanacaste, y que para el año 2018 reportan como lugar de residencia las provincias de San José y Heredia, lo que 
da cuenta de la necesidad que tienen de residir en un lugar más cercano a la Universidad.

Ello se hace evidente cuando se analizan los cantones de cada una de las provincias de residencia del estudiantado, donde los cantones centrales son los más frecuentes. Mientras que existe una mayor diversidad de cantones cuando se trata de las provincias de origen, ya que se incluyen otros más alejados del centro de estas, tendencia que se mantiene.

\section{CARACTERÍSTICAS SOCIOECONÓMICAS}

En lo que respecta a la dimensión económica, se encuentra que la mayoría del estudiantado depende económicamente de alguien, sean padres $u$ otros familiares, o bien de la beca de la Universidad, correspondiendo a un total de $66 \%$ de la población.

Cabe aclarar que se les consultó sobre la principal fuente de ingreso económico, por lo que no se descarta que puedan existir combinaciones entre las opciones que se les dieron, por ejemplo, que requieran de la ayuda económica de algún familiar junto con la beca que la Universidad les brinda, o bien, que realicen algún trabajo remunerado que les proporcione algún ingreso, aunque no el suficiente, $y$ que requieran de ayuda familiar o de la beca.

\section{GRÁFICO 1 \\ PRINCIPAL FUENTE DE INGRESO ECONÓMICO \\ DEL ESTUDIANTADO DE LA CARRERA DE SOCIOLOGÍA \\ UNIVERSIDAD DE COSTA RICA, 2018}

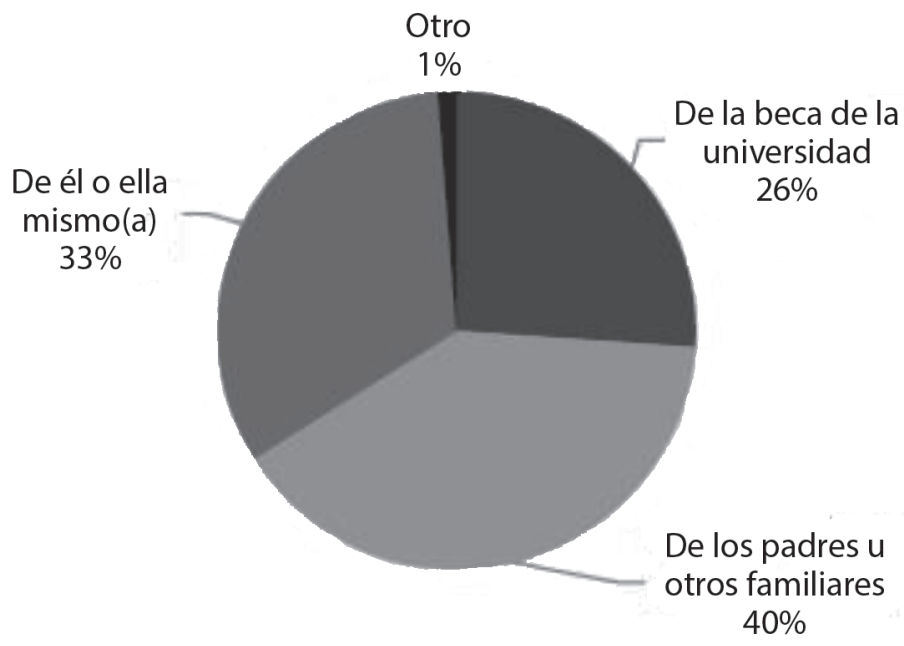

Fuente: Elaboración propia a partir de los datos del cuestionario aplicado al estudiantado activo de la carrera de Sociología durante el ciclo I-2018.

Como se muestra en el gráfico 1, el $40 \%$ depende económicamente de sus padres $\mathrm{u}$ otro familiar, mientras que un $33 \%$ depende de sí mismo o de sí misma. Por su parte, un $26 \%$ depende principalmente de la beca de la Universidad y el $1 \%$ señala que tiene otras fuentes de ingreso como la pensión o las horas de asistencia en la Universidad.
Asimismo, se le consultó a la población estudiantil si realizaban algún tipo de trabajo remunerado, con independencia de si dependen o no exclusivamente de este para subsistir. Del total de las respuestas registradas, el 39\% indicó que sí trabaja, correspondiendo a 187 estudiantes. Del total del estudiantado que labora, no existe una diferencia significativa por 
motivo de género. Sin embargo, en esta variable se invierte la prevalencia del género femenino.

Se destaca que del porcentaje de estudiantes que trabajan, solo el 30\% (57 estudiantes) realizan labores vinculadas con la Sociología. Dentro de las tareas que realizan se encuentran, por ejemplo: análisis de datos cuantitativos y cualitativos (recolección de información e interpretación), gestión de proyectos (evaluaciones y diagnósticos), promoción social, en temas de migrantes, empoderamiento de grupos vulnerables y desarrollo humano; así como, estudios de mercado y docencia universitaria. Mientras que el $70 \%$ restante labora en actividades no vinculadas con la Sociología, donde destacan trabajos vinculados con otras carreras que han completado previamente, como la de Derecho o la de Ciencias Políticas. Además de otras labores relacionadas con los call center, trabajo por cuenta propia, dependiente en diversos comercios, diseño gráfico, entre otras.

De esta población, más de la mitad labora tiempo completo, mientras que solo un $11 \%$ trabajan más de 24 horas, pero menos de tiempo completo (ver gráfico 2).

\section{GRÁFICO 2 \\ CANTIDAD DE HORAS SEMANALES QUE LABORA LA POBLACIÓN ESTUDIANTIL DE LA CARRERA DE SOCIOLOGÍA QUE REALIZA TRABAJO REMUNERADO 2018}

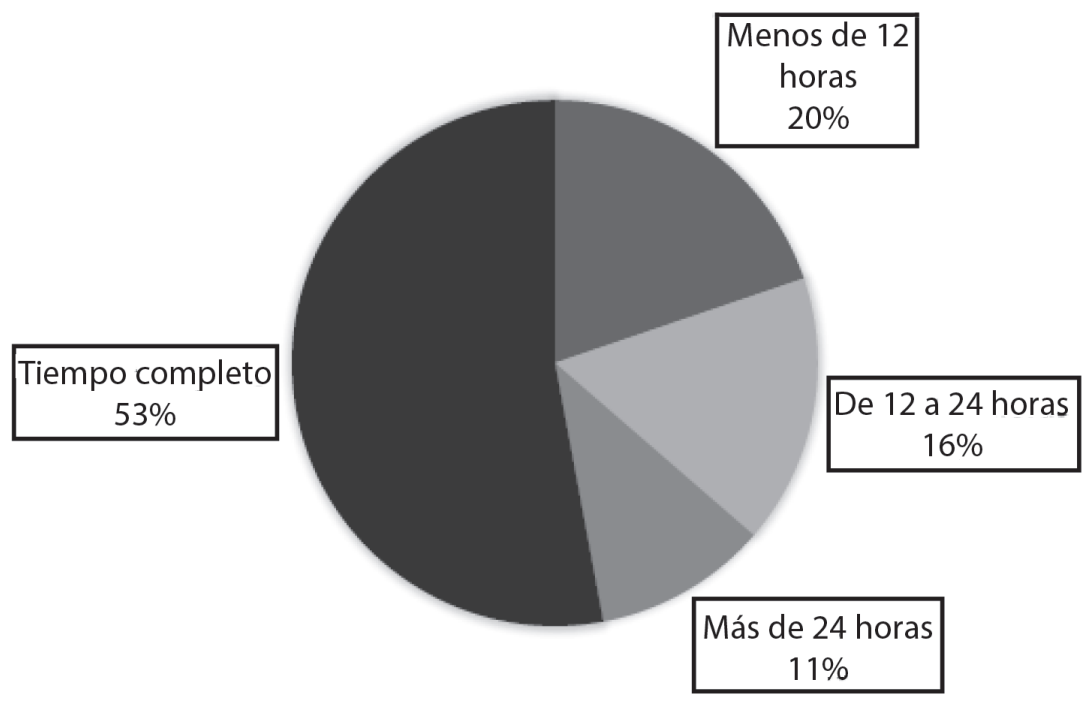

Fuente: Elaboración propia a partir de los datos del cuestionario aplicado al estudiantado activo de la carrera de Sociología durante el ciclo I-2018.

Si bien, prevalece la población que realiza labores no vinculadas con la Sociología, para quienes realizan trabajos sociológicos se mantiene una prevalencia del tiempo completo en el $61 \%$ del estudiantado $y$, en segundo lugar, se ubican quienes laboran menos de 12 horas, que corresponden al $16 \%$.
Esta información es de suma importancia para las unidades académicas, ya que se debe tener en consideración al grupo de la población estudiantil que trabaja y que además lo hace en su mayoría tiempo completo. Del porcentaje de estudiantes que trabajan a tiempo completo, un $57 \%$ tiene un horario diurno. 
Con el fin de tener un panorama amplio de las condiciones socioeconómicas del estudiantado, se indagó sobre el nivel educativo y la ocupación de ambos progenitores del estudiantado, al tener en consideración que la mayor parte de la población estudiantil depende económicamente de sus padres.

Al respecto, cabe destacar en términos generales, que el nivel educativo de los padres es ligeramente mayor que el nivel de las madres del estudiantado, aunque la diferencia no es significativa, la misma existe. Esta desigualdad se observó sobre todo en el grado Universitario con título o completo, con un $30 \%$ para las madres y un $32 \%$ para los padres. Otro elemento a considerar, es que las madres cuentan con una mayor prevalencia en lo que respecta a la segundaria completa y en la formación de estudios técnicos.

Con respecto a la ocupación de las madres y de los padres, se observó que las actividades más frecuentes entre las madres son las labores domésticas (46\%), seguida de labores profesionales $y$ de actividades vinculadas con el comercio, la venta y los servicios directos. Por su parte, en lo que refiere a los padres, la categoría de "otro" es la más prevalente (19\%), donde se agrupan labores como los desempleados, policías y agentes de seguridad, entre otras; también, en este porcentaje se agrupa la población que no conoce o no tiene contacto con su padre. A esta le siguen las ocupaciones relacionadas con la construcción, la mecánica, el transporte y la operaria, así como, las labores profesionales (14\%) y las vinculadas con el comercio, ventas y servicios directos. Destaca que las labores de dirección y gerencia son poco significativas entre la totalidad de las actividades. Sin embargo, a pesar de ello existe diferencia entre los padres y las madres, al ser más frecuente entre los primeros que en las segundas este tipo de labores.

Se evidencia que a pesar de que los progenitores cuentan con un nivel educativo, que se puede considerar alto, a partir del análisis de los datos no se puede afirmar que la mayoría cuenta con un nivel socioeconómico alto. Asimismo, la mayoría es asalariado o asalariada. Lo anterior adquiere una mayor relevancia cuando se recuerda que el $40 \%$ del estudiantado depende económicamente de ellos u otros familiares.

\section{CARACTERÍSTICAS ACADÉMICAS}

Esta dimensión se caracteriza por ser de mucha importancia en la configuración del perfil del estudiantado activo, ya que da cuenta de la forma en que la población estudiantil completa el Plan de Estudios, y permite relacionar con mayor claridad las dos dimensiones desarrolladas previamente.

El punto de partida para la comprensión del estudiantado en la carrera, fue determinar la condición del estudiante activo o activa, ello supone determinar y definir las categorías en las cuales se encuentran en el Plan de Estudios. Al respecto, se determinaron 7 condiciones, a partir de las cuales se logró establecer una clasificación del estudiantado (ver tabla 2). 
TABLA 2

CONDICIÓN DEL ESTUDIANTADO ACTIVO EN LA ESCUELA DE SOCIOLOGÍA

UNIVERSIDAD DE COSTA RICA, 2018

\begin{tabular}{l|cc}
\hline CONDICIÓN DEL ESTUDIANTADO ACTIVO & $\begin{array}{c}\text { VALORES } \\
\text { ABSOLUTOS }\end{array}$ & PORCENTAJE \\
\hline $\begin{array}{l}\text { Se encuentra cursando el bachillerato, y matriculó } \\
\text { cursos del plan de estudios }\end{array}$ & 312 & $50 \%$ \\
$\begin{array}{l}\text { Se encuentra cursando el bachillerato, pero no matriculó } \\
\text { cursos del plan de estudios }\end{array}$ & 173 & $8 \%$ \\
Otro & 52 & $4 \%$ \\
$\begin{array}{l}\text { Ya es egresado(a) de licenciatura y se encuentra realizando } \\
\text { su trabajo final de graduación }\end{array}$ & 24 & $4 \%$ \\
$\begin{array}{l}\text { Ya es egresado(a) del bachillerato, y se encuentra llevando } \\
\text { cursos de la licenciatura }\end{array}$ & 23 & $3 \%$ \\
$\begin{array}{l}\text { Ya es egresado(a) de licenciatura, pero no ha iniciado su } \\
\text { trabajo final de graduación }\end{array}$ & 21 & $2 \%$ \\
$\begin{array}{l}\text { Ya es egresado (a) del bachillerato, pero no matriculo cursos } \\
\text { de la licenciatura }\end{array}$ & 15 & $1 \%$ \\
$\begin{array}{l}\text { Se encuentra llevando cursos pendientes del bachillerato, } y \\
\text { también está adelantando curso de la licenciatura }\end{array}$ & 9 & $100 \%$ \\
\hline \begin{tabular}{l} 
TOTAL \\
\hline
\end{tabular}
\end{tabular}

Fuente: Elaboración propia a partir de los datos del cuestionario aplicado al estudiantado activo de la carrera de Sociología durante el ciclo I-2018.

Como se aprecia en la tabla 2 , la condición más frecuente y que representa el $50 \%$ corresponde al estudiantado que cursa el nivel de bachillerato y que cuenta con cursos matriculados al momento de la aplicación del instrumento. La segunda condición más recurrente es la población que también se encuentra llevando el bachillerato, pero que al momento del estudio no matriculó ningún curso (28\%). Posteriormente, se encuentran quienes indicaron la categoría de otro (8\%), donde la totalidad de las personas señalaron que no tener interés en continuar con la carrera. Solo el 1\% llevan cursos del bachillerato y a su vez adelantan cursos de la licenciatura. El porcentaje restante corresponde a los egresados y las egresadas, donde el $4 \%$ indicó que finalizó el plan de estudio de la licenciatura y esta en proceso de concluir su trabajo final de graduación, mientras que otro $4 \%$ del estudiantado finalizó el bachillerato, y llevan cursos de licenciatura. Por último, el $5 \%$ restante corresponde al estudiantado que siendo egresado o egresada de la licenciatura, no han iniciado el trabajo final de graduación (2\%), o quienes finalizaron el bachillerato y no han iniciado la licenciatura (3\%).

Es claro que la mayor parte del estudiantado activo de la Escuela, se encuentra cursando el bachillerato, del total de 629 estudiantes que indicaron su condición, 421 estudiantes (67\%) lo está completando y desean finalizarlo. Es importante indicar que las personas estudiantes que indicaron la categoría de "otro" (52 estudiantes), señalaron no querer continuar con la carrera de Sociología: la mayoría indicó la falta de interés en esta, o bien, decidieron 
cursar otra que les permitiera ingresar al mercado laboral de mejor manera. Asimismo, 73 estudiantes que no matricularon cursos del bachillerato y 2 estudiantes que siendo egresados del bachillerato no matricularon cursos de la licenciatura, también señalaron ya no tener más interés en la carrera.

Del total de 188 estudiantes que no matricularon en el semestre del estudio, tanto quienes cursan el bachillerato como las personas egresadas, que no matricularon cursos de la licenciatura, se puede indicar que el $60 \%$ (113 estudiantes) señaló sí tener interés en continuar con la carrera, pero la principal razón para no matricular es estar llevando otra carrera universitaria, la cual era su prioridad $y$ por lo cual le impedía llevar cursos de Sociología. Como segunda razón en orden de importancia, es el trabajo, puesto que señalan que los horarios son variables y laboran tiempo completo, por lo que esto les impide poder llevar los cursos. Además, se ubica en tercer lugar la dificultad de matricular los cursos pendientes porque los horarios no se adaptan a sus necesidades, $y$, por último, la falta de tiempo. No se encontró una diferencia significativa por género.

Del total del estudiantado activo con interés en completar la carrera, se encontró que se replica la diferencia por género indicada en los aspectos demográficos, en lo que respecta a cada una de las condiciones del estudiantado activo. No siendo significativa esta distinción en las categorías construidas, prevalece el femenino sobre el masculino.

Además, se interesó indagar por el tipo de colegio de procedencia; de los 502 estudiantes, el $72 \%$ del estudiantado activo realizó sus estudios secundarios en un colegio público y el restante $28 \%$ cursó la secundaria en un colegio privado.

En esta misma línea, un aspecto relevante fue consultar al estudiantado, cuál fue el interés que mostraron por la Sociología al momento de ingresar a la Universidad, en tanto si esta fue o no su primera opción como carrera en el proceso de ingreso. Al respecto, se detalla en la tabla 3 .

TABLA 3

SITUACIÓN DE INGRESO DEL ESTUDIANTADO A LA CARRERA DE SOCIOLOGÍA UNIVERSIDAD DE COSTA RICA, 2018

\begin{tabular}{|c|c|c|}
\hline INGRESO A LA CARRERA & $\begin{array}{l}\text { VALORES } \\
\text { ABSOLUTOS }\end{array}$ & PORCENTAJE \\
\hline Su segunda opción de carrera & 134 & $29 \%$ \\
\hline Su primera opción de carrera & 123 & $26 \%$ \\
\hline $\begin{array}{l}\text { Hizo solicitud de traslado para llevar la carrera de Sociología } \\
\text { de manera conjunta con su primera opción }\end{array}$ & 97 & $21 \%$ \\
\hline $\begin{array}{l}\text { Hizo solicitud de traslado por no interesarle la carrera a la } \\
\text { que había ingresado }\end{array}$ & 92 & $20 \%$ \\
\hline Segunda carrera, luego de haber finalizado la primera & 20 & $4 \%$ \\
\hline TOTAL & 466 & $100 \%$ \\
\hline
\end{tabular}

Fuente: Elaboración propia a partir de los datos del cuestionario aplicado al estudiantado activo de la carrera de Sociología durante el ciclo I-2018. 
Como se observa en la tabla 3 , la mayoría señaló que la Sociología fue su segunda opción de ingreso, y se quedaron en la carrera porque les comenzó a interesar, o bien, porque no lograron trasladarse a su primera opción. Asimismo, con una frecuencia similar, se encuentra el grupo de estudiantes que indicaron que la Sociología fue su primera opción. Por su parte, el $41 \%$ restante corresponde a quienes hicieron solitud de traslado.

Dentro de las razones señaladas por las personas que estudian Sociología para seleccionar esta carrera, se encuentran:

$\diamond \quad$ Interés personal y profesional: el $90 \%$ (416 estudiantes) mencionan que el principal motivo por el que estudian Sociología es la afinidad temática que tiene la disciplina con sus vidas e historias (indican que es una pasión, que les "gustan" los contenidos analizados $y$ les "encanta" trabajar con la gente). También señalan que la carrera les permite desarrollar el interés que tienen en la comprensión del comportamiento humano en sociedad, las múltiples dinámicas colectivas y la interacción social.

$\diamond \quad$ Complemento para la formación profesional: esta es la segunda razón más frecuente $(7 \%)$. Si bien, mencionan algunos aspectos destacados en el punto anterior, la razón más fuerte para estudiar Sociología fue el cubrir vacíos en la formación de su otra carrera. Indican claramente que es un complemento $y$ que les permite ampliar su conocimiento sobre lo social. Las carreras que llevan y que buscan apoyo en la Sociología para su formación y en el espacio profesional son: derecho, educación, trabajo social, comunicación colectiva, ciencias políticas, artes plásticas, entre otras. $\diamond \quad$ Referencias de otras personas: esta es la tercera razón más frecuente $(2 \%)$. Señalan que su interés por la carrera surge por influencia y socialización de docentes de secundaria, familiares y amigos. Si bien, comparten los temas sociológicos, fue la perspectiva de estos grupos, quienes los y las motivaron a comprender la importancia de la Sociología, con lo cual les llamó la atención ingresar a la carrera.

$\diamond \quad$ "No sé": esta es la cuarta razón que se logró registrar, corresponde al 1\% del estudiantado que indica no saber por qué estudia Sociología. Sin duda, esta no fue su primera opción de carrera, ingresaron $y$ no saben por qué siguen en esta. No indican mayores detalles en su respuesta.

Lo anterior se relaciona claramente cuando se les consulta si cursan otra carrera universitaria junto con Sociología, al respecto, el 59\% (275 estudiantes) solo llevan la carrera de Sociología, mientras que el 41\% (191 estudiantes) sî cursa otra carrera de forma conjunta.

Sin duda, la diversidad de carreras es amplia, donde se encuentran representadas casi todas las áreas de formación, desde Ciencias Sociales, Letras, Salud, Ingenierías, Educación, Ciencias Económicas, Agroalimentarias. Entre las más frecuentes se encuentran Educación e Ingeniería, aunque no de manera significativa frente a la Administración, el Derecho y las Ciencias Políticas. De los 191 estudiantes que estudian alguna de las carreras mencionadas, la mayoría, el $77 \%$, la cursa también en la Universidad de Costa Rica, mientras que el restante $23 \%$ se encuentra representado por las Universidades Privadas (10\%) y por las otras Universidades Públicas (13\%).

Asimismo, con respeto al nivel de carrera que cursan, se encuentra lo señalado en la tabla 4. 
TABLA 4

NIVEL DE LA CARRERA DE SOCIOLOGÍA DEL ESTUDIANTADO ACTIVO

UNIVERSIDAD DE COSTA RICA, 2018

\begin{tabular}{l|cc}
\hline NIVEL DE CARRERA & $\begin{array}{c}\text { VALORES } \\
\text { ABSOLUTOS }\end{array}$ & PORCENTAJE \\
\hline Introductorio (1 año) & 100 & $21 \%$ \\
Formativo (2 año) & 108 & $23 \%$ \\
Formativo (3 año) & 120 & $26 \%$ \\
Práctico (4 año) & 59 & $8 \%$ \\
Licenciatura (5 año) & 39 & $9 \%$ \\
Egresado(a) de licenciatura con el Trabajo Final de & 40 & $100 \%$ \\
\hline Graduación pendiente & 466 & 9 \\
\hline TOTAL & & \\
\hline
\end{tabular}

Fuente: Elaboración propia a partir de los datos del cuestionario aplicado al estudiantado activo de la carrera de Sociología durante I-2018.

Con respecto a la diferenciación de los niveles de carrera por rangos de edad, se encuentra que existe una correlación entre el nivel y el rango, donde a menor nivel de carrera menor es el rango de edad en el que se ubica el estudiantado, $y$, por el contrario, a mayor nivel de carrera mayor es la edad del estudiantado.

En lo que respecta a la forma en que la población estudiantil lleva o completa la carrera, se encontró los siguientes aspectos a destacar:

$\diamond \quad$ El primero refiere a que la mayoría del estudiantado indica que no puede llevar los bloques completos propuestos en el Plan de Estudios vigente, correspondiendo a este grupo el $63 \%$ (294 estudiantes). Dentro de las razones que señalan, la principal es laboral, donde la dificultad de hacer coincidir los horarios de clase con el trabajo impide que lleven la totalidad de los cursos requeridos en cada semestre. La segunda razón de importancia es que Sociología no es su carrera prioritaria, por lo que se encuentran llevando bloques completos de otra carrera y solo llevan algunos cursos de Sociología cada semestre. La tercera razón más frecuente, es la existencia de un promedio ponderado bajo que les impide encontrar cupo en todos los cursos. La cuarta razón también significativa y relacionada con el Plan de Estudios, es que mencionan que no pueden cumplir con la carga de los cursos, debido a que la misma es excesiva.

$\diamond \quad$ En relación con lo anterior, al consultarles por el número de créditos que llevan cada semestre en Sociología, la mayor parte de la población estudiantil (44\%) lleva entre 10 y 15 créditos por semestre, mientras que el $19 \%$ lleva entre 5 y 10 créditos, y el $18 \%$ entre 15 y 20 créditos por ciclo. Datos que resultan coincidentes con la dificultad que tiene la mayoría de completar los bloques completos.

$\diamond \quad$ Otra temática de interés en la dimensión académica, fue indagar en el conocimiento que tiene el estudiantado en 
un segundo o tercer idioma. Al respecto, el $77 \%$ (360 estudiantes) indicó tener conocimiento en otro $u$ otros idiomas diferentes del español, por su parte el $23 \%$ (106 estudiantes) indicaron no tener ningún tipo de conocimiento. Del total de 360 estudiantes (77\%) que indicaron tener conocimiento en otros idiomas, entre los más frecuentes que manifestaron saber se encuentran: inglés, portugués, francés, italiano y alemán. Sin embargo, estos no son los únicos, ya que también mencionaron conocer japonés, mandarín, coreano, árabe, ruso, catalán, indonesio, holandés, griego, chino, sueco, danés y esperanto (lengua planificada).

$\diamond \quad$ Cabe destacar al respecto de los idiomas que, aunque la mayoría no tiene dominio completo en alguno, una importante cantidad de estudiantes tiene conocimiento intermedio y avanzado.

Por último, para esta dimensión académica se les consultó sobre el interés que tienen en completar tanto el grado de licenciatura, como la maestría en Sociología. Sobre la licenciatura, la mayoría indicó tener interés en completarla correspondiendo al $82 \%$ (382 estudiantes), mientras que el $61 \%$ (264 estudiantes) indicó también completar el posgrado en Sociología. Esto estaca la importancia que tiene la carrera en el desarrollo de su formación profesional.

\section{ASPIRACIONES PROFESIONALES}

Con respecto a esta dimensión, se interesó en la fase cuantitativa, con el fin de recuperar la percepción general que tiene el estudiantado de su futuro profesional. Los datos que a continuación se describen permiten tener una mirada amplia de sus aspiraciones $y$ del papel que juega en la actualidad la Sociología en ese proyecto. Resultó importante recuperar la subjetividad colectiva, por lo que se sistematizó la información de manera que diera cuenta de las principales impresiones de cómo visualizan la Sociología en el ámbito profesional.

Por ello, se consultó al estudiantado sobre su principal objetivo profesional, con una amplia mayoría del $49 \%$ que señaló como objetivo el encontrar un trabajo estable $y$ vinculado con sus temáticas de interés sociológico. En segundo y tercer lugar, con una frecuencia similar correspondiendo al $20 \%$ cada objetivo, se encontró con las aspiraciones de especializarse en posgrados en el país o en el extranjero, así como encontrar un espacio laboral que les permita relacionar las carreras que estudiaron o que estudian, lo que es coincidente con el importante grupo de estudiantes que cursa junto con Sociología otra carrera, o bien, Sociología como segunda carrera. No menos importantes, pero si menos frecuentes, indican como su principal objetivo profesional convertirse en investigador e investigadora, generar impacto social con su formación en Sociología, o bien, mejorar sus condiciones laborales al obtener la titulación. Con ello, en la tabla 5 se muestra el posicionamiento del estudiantado según género. 
TABLA 5

POSICIONAMIENTO DEL ESTUDIANTADO SOBRE LA CARRERA DE SOCIOLOGÍA DENTRO

DEL MERCADO LABORAL SEGÚN GÉNERO

UNIVERSIDAD DE COSTA RICA, 2018

\begin{tabular}{l|ccc|c}
\hline POSICIÓN DE LA CARRERA EN EL FUTURO LABORAL & FEMENINO & MASCULINO & NO-BINARIO & TOTAL \\
\hline Es como cualquier otra, con ventajas y dificultades & 140 & 118 & 4 & 262 \\
Pocas oportunidades laborales & 92 & 51 & & 143 \\
Muchas oportunidades laborales & 10 & 7 & & 17 \\
Otro & 7 & 7 & & 14 \\
\hline TOTAL & 249 & 183 & 4 & 436 \\
\hline
\end{tabular}

Fuente: Elaboración propia a partir de los datos del cuestionario aplicado al estudiantado activo de la carrera de Sociología durante el ciclo I-2018.

En lo que respecta a los espacios laborales en los que desean insertarse, se encuentra que la mayoría quiere trabajar en el Estado, sea en el gobierno central o en las instituciones autónomas (34\%). Le sigue en orden de importancia, las entidades y los organismos no gubernamentales (17\%), así como en docencia e investigación en centros de educación superior (15\%), mientras que el $11 \%$ señaló querer laborar en organismos internacionales. Por su parte, el porcentaje restante muestra interés en realizar trabajos por cuenta propia (consultorías), laborar con organizaciones comunales $y$ locales, empresas privadas $y$ proyectos microempresariales o cooperativos. Asimismo, un $7 \%$ mencionó no tener una preferencia clara por alguna de las opciones indicadas, por lo que todas son de su interés o la necesidad de encontrar empleo los llevaría a aceptar cualquier opción que se les presente. Datos que son coincidentes con el principal objetivo profesional indicado por el estudiantado, cuando mencionaron que esperan encontrar trabajo estable.

Por último, en esta dimensión, y a partir de lo señalado por el estudiantado, se intentó conocer cuáles consideran que son los principales factores que inciden en la búsqueda de trabajo en Sociología. Al respecto, señalaron cinco elementos que consideran clave en su inserción en el mercado laboral, $y$ de los cuales llama la atención que los tres primeros aspectos los vinculan con herramientas que debe brindar la formación en la carrera, mientras que los dos últimos, son aspectos que se atribuyen al propio estudiante $y$ al contexto nacional.

$\diamond \quad$ El primer factor que señalan es la necesidad de contar con experiencia en las labores profesionales de la carrera fuera de las aulas. Se indica que se deben hacer pasantías o prácticas en instituciones $o$ en organismos no gubernamentales, tanto en investigación como en acción social. Esto desde los primeros años de carrera, al no considerar suficiente los talleres del nivel práctico (4 año). Mencionan que dichos talleres se comprenden como un curso más de la carrera $y$ no están pensados en ser experiencias laborales.

$\diamond \quad$ En segundo lugar, indican la ausencia de redes de contactos con otros $y$ otras profesionales. Se indica la necesidad de contar con mayor conocimiento de quiénes son las personas que laboran en ciertas áreas de la Sociología, quienes al momento de insertarse en el mercado laboral puedan ser de apoyo e información sobre las prácticas y tendencias sociológicas en el momento. 
$\diamond \quad$ El tercer aspecto, es la falta de un conocimiento real del mercado laboral. Se indica que, a lo largo de la formación, no se cuentan con espacio de análisis del mercado laboral en Sociología, cuál es su dinámica, dónde se labora, qué trabajos realizan, en cuáles temas. Ello como una contextualización de la realidad a la que se tendrán que enfrentar.

$\diamond \quad$ Para cada uno de estos tres elementos, indican que la Escuela de Sociología debe ser la encargada de brindar estos espacios de conocimiento $y$ formación, con el fin de preparar al estudiantado para el mercado laboral. No por ello, aclaran, se debe adecuar la carrera al mercado, pero sí reclaman la necesidad de conocer el espacio laboral al que tendrán que enfrentarse.

$\diamond \quad$ Como cuarto elemento, mencionan la necesidad de contar con conocimientos en otros idiomas como una herramienta importante en la incorporación al mercado laboral, al ser esto indispensable en el contexto actual, y lo cual permitiría la inclusión de la Sociología en distintos espacios y mercados.

$\diamond \quad$ Por último, indican que un factor crucial es la necesidad de ampliar el mercado laboral sociológico. Rescatan la importancia de que los empleadores conozcan más sobre la carrera, sobre el perfil de profesionales que se forman, con el fin de abrir nuevos espacios que permitan una incorporación al ámbito laboral menos complicada y con mayores opciones de escogencia y crecimiento profesional.

\section{PROYECTO DE VIDA}

Esta dimensión, al igual que la anterior, intenta reunir las posiciones subjetivas del estudiantado, principalmente en lo que respecta a las vinculaciones que perciben entre la carrera y su vida futura. Asimismo, se interesó indagar dónde en su proyecto de vida está la Sociología y qué lugar ocupa; además de las acciones que esperan emprender para que este sea real. Esto porque se comprende al estudiantado dentro de un conjunto de relaciones sociales y culturales, donde la selección de la carrera es un aspecto importante.

Por ello, se les consultó sobre su proyecto de vida a corto plazo, en los próximos dos años. El estudiantado coincidió en siete proyectos concretos que espera alcanzar. Destaca que la gran mayoría del estudiantado vincula su proyecto de vida con la carrera. El $87 \%$ señala proyectos vinculados con la Sociología, de este porcentaje, el $45 \%$ tiene como propósito avanzar en la carrera, el $36 \%$ graduarse, mientas que el $6 \%$ indica buscar vinculaciones con instituciones para adquirir experiencia. Por su parte, el $13 \%$ restante menciona encontrar trabajo, aprender un segundo idioma, realizar pasantías y estudiar en el extranjero.

En lo que respecta a sus proyectos de largo-mediano plazo (5 años), resaltan dos aspectos: 1) que el número de proyectos se homogeniza, y 2) que la carrera de Sociología, a pesar de seguir teniendo protagonismo, ya no es el centro de la mayoría de los proyectos de vida del estudiantado. Se menciona como el más importante el graduarse, donde el $42 \%$ del estudiantado lo indica; así mismo, en segundo lugar, está el encontrar trabajo (38\%), el que adquiere un peso mayor en la frecuencia de estudiantes que lo mencionan; en tercer lugar, señalan el especializarse o iniciar otra carrera (14\%), lo que evidencia su interés en continuar con su formación profesional; $y$, por último, un $6 \%$ indica interés en comprar algún tipo de bien mueble o inmueble.

Al respecto de la consulta sobre las acciones concretas que consideran y deben emprender para cumplir con sus proyectos de vida, indican las siguientes:

$\diamond \quad$ Terminar cursos pendientes.

$\diamond \quad$ Adquirir mayores habilidades técnicas (nuevos conocimientos acordes con el mercado laboral, metodológicos, más idiomas).

$\diamond \quad$ Planificación y organización de los recursos con los que cuenta.

$\diamond \quad$ Seguir estudiando, especializarse.

$\diamond \quad$ Adquirir experiencia en trabajos sociológicos y participar de proyectos.

$\diamond \quad$ Encontrar trabajo. 
$\diamond \quad$ Generar una red de contactos de colegas.

$\diamond \quad$ Mejorar las calificaciones.

$\diamond \quad$ Vivir bien (cuidar la salud física y mental).

Continúan siendo protagónicas las acciones concretas vinculadas con la carrera, lo que coincide con sus aspiraciones profesionales y su proyecto de vida. Sin embargo, destaca la acción de "vivir bien", la cual fue mencionada por un amplio número de estudiantes.

Por último, se les consulta directamente sobre la relación de la Sociología con su proyecto de vida, a lo que sus respuestas son las siguientes:

$\diamond \quad$ "En todo", centro de su proyecto de vida.

$\diamond \quad$ Aporta a lo académico, es una parte del proyecto de vida.

$\diamond \quad$ Permite tener una perspectiva más amplia de la vida $y$ de los individuos al alrededor ("es una forma de mirar el mundo").

$\diamond \quad$ Complemento a otras carreras.

$\diamond \quad$ Herramienta para generar cambio.

$\diamond \quad$ Se vincula con la búsqueda de trabajo.

Las relaciones señaladas por el estudiantado dan cuenta de que una parte importante de la población comprende la Sociología como su proyecto de vida. Sin embargo, también permite ver otros niveles $y$ formas de vinculación.

\section{CONCLUSIONES}

El presente artículo busca que la información mostrada sea valiosa para el adecuado cumplimiento de las tareas y de los procesos de apoyo que la Escuela de Sociología debe llevar a cabo con el estudiantado en procura de su adecuada vinculación, formación, orientación académica $y$ profesional.

Este no es más que un primer acercamiento a las dimensiones mencionadas, lo que puede traducirse más adelante en algunas líneas investigativas para futuros proyectos, tanto en Sociología como en otras unidades académicas.

Resulta de importancia destacar la comprensión de un perfil del estudiantado amplio, que no solo comprenda dimensiones tradicionales, entiéndase la demográfica o socioeconómica, sino que contemple otras como la académica, las aspiraciones profesionales y el proyecto de vida. Destaca en estas últimas, la subjetividad y las experiencias que durante el proceso educativo tiene el estudiantado $y$, a partir del cual, construye sus significaciones $y$ vivencias.

En cuanto a la dimensión demográfica, se puede afirmar que el estudiantado se caracteriza por ser en su mayoría una población joven, soltera, con una leve prevalencia del género femenino sobre los demás, costarricense, sin hijos o hijas, que aún viven con sus padres $\mathrm{u}$ otros familiares, $y$ que es originaria $y$ residente del GAM.

Mientras que, en la dimensión económica, la mayoría del estudiantado activo de la Escuela depende económicamente de sus padres u otros familiares, así como de la beca de la Universidad, lo que guarda relación con el bajo porcentaje de estudiantes que realiza una actividad renumerada. El nivel educativo de los progenitores se caracteriza por ser alto, donde más del $50 \%$ cuenta con secundaria completa y más, siendo las labores domésticas la principal ocupación de las madres y en el caso de los padres no existe una actividad que prevalezca; ambos grupos se caracterizan por ocupar la posición de asalariados.

Asimismo, se puede indicar que como características principales en la dimensión académica se tiene un conjunto importante de estudiantes que desean completar la carrera, a pesar de que para la mayoría esta no fue su primera opción de ingreso a la Universidad. Se cuenta con un importante porcentaje del estudiantado que proviene de una formación en secundaria pública. Además, la población estudiantil se concentra en los primeros tres años de carrera, siendo difícil para la mayoría llevar los bloques completos en cada ciclo, principalmente por sus compromisos laborales y los vinculados con la otra carrera universitaria que llevan de manera conjunta. Tienen conocimiento en otros idiomas, donde el que impera es el inglés. Asimismo, reconocen la importancia de la disciplina en sus vidas personales y académicas, por lo que destaca el interés que manifiestan tener en 
continuar con la licenciatura y con la maestría de la Escuela. Cabe subrayar que quienes no matricularon cursos, tienen otras prioridades que les impiden llevar de manera regular la carrera, tal y como se indicó por sus situaciones de estudio, laborales, económicas, familiares o de salud, la Sociología no es su prioridad. A pesar de lo anterior, continúan teniendo interés por la carrera y reconocen la importancia de esta en su formación.

Si bien, el porcentaje de personas que ingresan a la carrera por interés es importante, existe un grupo también significativo que la llevan porque no ingresaron a su primera opción. Esto recuerda la relevancia que tienen los cursos del nivel introductorio, en tanto permitan captar esa población estudiantil no segura de estudiar Sociología, o bien, que los cursos les brinden la información necesaria para que tomen la decisión de dejarla y hacer la gestión de traslado a otra.

Sin duda, la mayoría percibe su futuro de manera cautelosa, con lo cual se evidencia una mirada clara de la realidad para analizar las posibilidades existentes en el marco de las actuales características del mercado laboral, donde se compite con otras ciencias sociales $y$ se legitiman otras carreras; pero que, a pesar de ello, existen espacios sociológicos para su inserción laboral. De ahí que sus aspiraciones profesionales se resumen en encontrar trabajo estable y sociológico, principalmente en el Estado y sus instituciones, así como en organizaciones no gubernamentales. Se debe considerarque la mayoría creen que la carrera de Sociología es como cualquier otra, con sus ventajas $y$ dificultades en la inserción laboral; sin embargo, destacan factores en su formación que deben existir para que ese proceso sea mejor y más fácil.

Cabe señalar que existe una clara coincidencia entre las dimensiones de aspiraciones profesionales y los proyectos de vida, lo que es claro cuando se recuerdan las características demográficas y socioeconómicas de las personas estudiantes que cursan la carrera.

Aunado a ello, y luego de la presentación de los principales resultados y caracterización del perfil del estudiantado, se considera que es innegable la relevancia que este tipo de información tiene o podría tener para una Escuela, tanto para el profesorado y para el estudiantado, como para sus Comisiones permanentes y el área administrativa.

Es importante subrayar que son más los aportes que las limitaciones que este tipo de práctica puede tener. Al respecto, este tipo de estudio sirve de apoyo en las gestiones académico-administrativas identificadas en la unidad académica, específicamente en el análisis de la estructura del Plan de Estudio, en la planificación de la propuesta de horarios de cada ciclo lectivo, al profesorado en la compresión de la población estudiantil que tiene en cada una de sus clases, así como prestar atención a la importancia de la formación complementaria requerida por el estudiantado para su incorporación en el mercado laboral.

En suma, este tipo de caracterizaciones deberían ser más frecuentes en las unidades académicas de la Universidad, ya que es claro su importante potencial y contribución en la toma de las decisiones, al ser un punto de partida informado $y$ contextualizado.

A partir de este hecho, la Universidad no transmite información, sino que es facilitadora en ese proceso de construcción del conocimiento, a partir del debate, la discusión $y$ la criticidad de los diversos contenidos que se abordan en los diferentes cursos de las carreras, lo que contribuye en la optimización del proceso y quehacer educativo, además de facilitar los propósitos de bienestar estudiantil que tiene la universidad pública.

\section{REFERENCIAS}

Caira, N. y Lescher, I. (2013). Perfil del estudiante de Sociología de la Universidad del Zulia. Revista de Ciencias Sociales, XIX (3), 588-600.

Carreño, B., Micin, S. y Urzua, S. (2016). Una caracterización inicial para el logro académico de estudiantes de primer año universitario. Cuadernos de Investigación Educativa, 7(1), 29-39.

Centro de Investigaciones. Departamento de Bienestar Universitario. (2005). Caracterización de los estudiantes de una 
Institución de Educación Superior de Pereira. Investigaciones Andina, 7 (10), 10-16.

Cruz, S. y Rodríguez, J. (2012). Pistas de quiénes son y cómo aprenden los estudiantes de la Escuela de Ciencias de la Comunicación Colectiva de la Universidad de Costa Rica. Revista Reflexiones/Jornadas de Investigación, 33-43.

De Sousa, B. (2007). La universidad en el siglo XXI: para una reforma democrática $y$ emancipadora de la universidad. CIDESUMSA, ASDI y Plural Editores.

Garay, A. (2013). El perfil de los estudiantes de nuevo ingreso de las universidades tecnológicas en México. El Cotidiano, 19 (122), 75-85.

Garza, L., Contreras, R. y Ruiz, M. (2013). Perfil de ingreso $y$ factores de riesgo y protección de estudiantes de una dependencia de educación superior. Revista de Psicología y Ciencias del Comportamiento, 4 (1), 47-58.

Molina, E. (2018). Aproximaciones en el campo de la enseñanza-aprendizaje de la investigación social en la Escuela de Sociología de la Universidad de Costa Rica. Informe Final de Investigación, Proyecto 01-1411-2016. Escuela de Sociología, Universidad de Costa Rica.

Plan de Estudios de la Licenciatura (2018). Escuela de Sociología, Universidad de Costa Rica.

Plan de Estudios del Bachillerato (2016). Escuela de Sociología, Universidad de Costa Rica.

Rodríguez, J. y Rojas, X. (2015). Perfil de los estudiantes de bibliotecología y ciencias de la información en la Universidad de Costa Rica. e-Ciencias de la Información, 5(2), 1-23.

Strauss, A. y Corbin, J. (2002). Bases de la investigación cualitativa: técnicas y procedimientos para desarrollar la teoría fundamentada. Editorial Universidad de Antioquia.

Fecha de ingreso: 20/11/2019 Fecha de aprobación: 13/11/2020 